\title{
3722
}

\section{Association between blood eosinophil count and exacerbation risk in patients with asthma receiving medium- or high-dosage inhaled corticosteroids}

\author{
Asthma - management, Exacerbation, Physiology
}

M. Kerkhof ${ }^{1 *}$, T. Tran ${ }^{2}$, M. van den Berge ${ }^{3}$, G. Brusselle ${ }^{4}$, G. Gopalan ${ }^{2}$, R. Jones $^{5}$, J. $^{2}$ Kocks $^{3}$, A. Menzies-Gow ${ }^{6}$, J. Nuevo ${ }^{2}$, I. Pavord ${ }^{7}$, S. Rastogi ${ }^{2}$, D. Price ${ }^{1}$

1Observational and Pragmatic Research Institute - Singapore (Singapore), ${ }^{2}$ AstraZeneca Gaithersburg, MD (USA), ${ }^{3}$ University of Groningen, University Medical Center Groningen Groningen (Netherlands), ${ }^{4}$ Ghent University Hospital - Ghent (Belgium), ${ }^{5}$ The Peninsula College of Medicine and Dentistry - Plymouth (United Kingdom), ${ }^{6}$ Royal Brompton and Harefield NHS Foundation Trust - London (United Kingdom), ${ }^{7}$ Nuffield Department of Medicine, University of Oxford - Oxford (United Kingdom)

Introduction: Patients with high blood eosinophil counts (BEC) may be at increased risk of asthma exacerbations (Lancet Respir Med. 2015;3:849-58).

Aims and Objectives: We studied whether high BEC were associated with more asthma exacerbations in patients receiving medium- or high-dosage ICS.

Methods: We analyzed 2 years of medical records data for 189,031 patients with asthma (aged $\geq 13$ years) receiving stable ICS therapy, from the Clinical Practice Research Datalink and the Optimum Patient Care Research Database. Patients' baseline data came from the year before their most recent blood eosinophil count. Number of exacerbations was counted in a follow-up year. Negative binomial regression was used to estimate the adjusted association between high BEC $\left(\geq 0.35 \times 10^{9} / \mathrm{L}\right)$ and exacerbation rate.

Results: $62,476(33 \%)$ and 32,728 (17\%) patients were receiving medium- or high-dosage ICS, respectively, and $26 \%$ in each group had high BEC. Nonsmokers receiving medium- or high-dosage ICS with high $\mathrm{BEC}$ had significantly greater exacerbation rates than those without high BEC (rate ratio: 1.36; $95 \% \mathrm{Cl}: 1.30-1.43)$. Associations were comparable for nonsmokers with high BEC whether they received medium- $(1.37 ; 95 \% \mathrm{Cl}$ : $1.29-1.45)$ or high-dosage $(1.32 ; 95 \% \mathrm{Cl}$ : $1.23-1.41)$ ICS. The association was significantly weaker in current smokers $(1.15 ; 95 \% \mathrm{Cl}$ : 1.07-1.23) and ex-smokers (1.24; 95\% Cl: 1.18-1.31). Similar associations were found in patients with good adherence (Medication Possession Ratio $\geq 80 \%$ ). 
http://abstract.ersnet.org/Front/Submission/PreviewAbstract?repository...

Conclusions: Patients with asthma and high BEC have an increased risk of exacerbations despite medium- and high-dosage ICS treatment and good adherence. 